\title{
The development of the mucosal immune system pre- and post-weaning: balancing regulatory and effector function
}

\author{
M. Bailey*, K. Haverson, C. Inman, C. Harris, P. Jones, G. Corfield, B. Miller and C. Stokes \\ School of Clinical Veterinary Science, University of Bristol, Langford House, Langford, Bristol BS4 5DU, UK
}

\begin{abstract}
The mucosal immune system fulfils the primary function of defence against potential pathogens that may enter across vulnerable surface epithelia. However, a secondary function of the intestinal immune system is to discriminate between pathogen-associated and 'harmless' antigens, expressing active responses against the former and tolerance to the latter. Control of immune responses appears to be an active process, involving local generation of $\mathrm{IgA}$ and of regulatory and/or regulated $\mathrm{T}$ lymphocytes. Two important periods of maximum exposure to novel antigens occur in the young animal, immediately after birth and at weaning. In both cases the antigenic composition of the intestinal contents can shift suddenly, as a result of a novel diet and of colonisation by novel strains and species of bacteria. Changes in lifestyles of man, and husbandry of animals, have resulted in weaning becoming much more abrupt than previously in evolution, increasing the number of antigens that must be simultaneously evaluated by neonates. Thus, birth and weaning are likely to represent hazard and critical control points in the development of appropriate responses to pathogens and harmless dietary and commensal antigens. Neonates are born with relatively undeveloped mucosal immune systems. At birth this factor may prevent both expression of active immune responses and development of tolerance. However, colonisation by intestinal flora expands the mucosal immune system in antigenspecific and non-specific ways. At weaning antibody to fed proteins can be detected, indicating active immune responses to fed proteins. It is proposed that under normal conditions the ability of the mucosal immune system to mount active responses to foreign antigens develops simultaneously with the ability to control and regulate such responses. Problems arise when one or other arm of the immune system develops inappropriately, resulting in inappropriate effector responses to harmless food proteins (allergy) or inadequate responses to pathogens (disease susceptibility).
\end{abstract}

Oral tolerance: Immune development: Weaning: Pig: Allergy

Although the majority of research on the function of the immune system has concentrated on responses to injected antigen, the major site of exposure to 'foreign' antigens is at body surfaces, of which, the largest antigenic load occurs in the intestine. In this site an absorptive epithelium one cell deep is exposed to macromolecules derived from food and from intact enteric bacteria and their metabolic products.

The permeability of this epithelium, necessary for absorption of nutrients, carries with it the risk of simultaneous absorption of 'foreign' antigens that are actually harmless. Similarly, the need to maintain a mucosal immune system capable of recognition of potential pathogens and expression of appropriate active immune responses carries with it the risk of mounting active immune responses to these antigens. Thus, the twin evolutionary drives, to acquire nutrients and to defend against pathogens, result in the potential for expression of active immune responses against harmless environmental antigens.

The expression of active immune responses against pathogens is frequently disadvantageous for an individual organism. Induction of responses, proliferation of appropriate cell types and synthesis and secretion of appropriate effector molecules require diversion of energy and resources from other systems. The effector mechanisms of immune responses frequently result in tissue inflammation and damage, independent of that

\footnotetext{
Abbreviation: TGF $\beta$, transforming growth factor $\beta$; Th, T-helper.

*Corresponding author: Dr M. Bailey, fax + 44117928 9505, email mick.bailey@bristol.ac.uk
} 
generated by the pathogen. Presumably, the temporary disadvantage of expression of immune responses outweighs the long-term disadvantage of having to live, or die, with the pathogen. Since the pathogenicity of microorganisms varies from severe (e.g. Vibrio cholerae) to low or absent (true commensal flora, food), there is also the need for an ability to modulate immune responses dependent on the perceived threat, independent of the antigenic load; i.e. the magnitude and type of response should be dependent on the 'quality' of the antigen, not only on the quantity. In the case of most food antigens, in normal individuals exposure would, ideally, involve the complete absence of immune responses, or 'tolerance'.

Clearly, therefore, the mucosal immune system must be able to discriminate in some way between antigens requiring active immune responses and those requiring tolerance. Somewhere in the immune system, or in its interaction with its supporting stromal environment, is machinery capable of selecting different pathways dependent on the type of antigen. Making the right selection is critical, since the wrong selection can result in allergic responses to food antigens or inadequate responses to pathogen.

\section{Recognition systems}

Importantly, this need to assess the 'quality' of antigens in addition to the quantity must have developed in parallel with the evolution of systems for recognition of foreign molecules. Such recognition systems are certainly present in all multicellular organisms studied and have probably always been linked to organ systems involved in the absorption of nutrients.

Broadly, mechanisms for the recognition of foreign macromolecules fall into two categories associated with innate and adaptive immune systems.

\section{Innate recognition systems}

All animal species studied express receptors capable of recognition of macromolecules not synthesised in that species and of initiation of defence reactions. In sponges (Porifera) lectins recognise microbial carbohydrate residues (Schroder et al. 2003). Insects also use lectins, as well as Toll-like receptors (Luo \& Zheng, 2000). In vertebrates the Toll-like receptors are well characterised and recognise a series of products such as bacterial lipopolysaccharide, flagellin and viral RNA (Beutler \& Rehli, 2002). However, vertebrates also use lectins such as the mannan-binding lectin that initiates the deposition of complement onto microbial surfaces expressing mannans (Teillet et al. 2005).

Such recognition systems are termed pattern-recognition receptors, since they bind to molecules expressed by a broad range of potentially-harmful organisms. Innate recognition systems probably arose polyphyletically, but the common feature is that the specificity of the recognition receptors is encoded in the germ-line gene, i.e. the genes for the receptors are inherited, expressed and passed on to the next generation with no greater level of change than any other genes. Thus, the value of novel specificities arising by gene duplication and/or mutation can be tested evolutionarily; if they are disadvantageous, they will be deleted.

\section{Adaptive recognition systems}

In contrast, adaptive immune systems generate specificities by genetic modification of the genes encoding the receptors. The adaptive immune system that is familiar, characterised by $\mathrm{B}$ and $\mathrm{T}$ lymphocytes, immunoglobulins, MHC molecules and T-cell receptors, arose very suddenly in the jawed fish (Litman et al. 1999). Although the genes encoding B- and T-cell recognition receptors are inherited and passed on unchanged, specificities are generated randomly in lymphocytes by a combination of genetic rearrangement and hypermutation. Importantly, the ability to express receptors capable of recognising random structures, including those associated with self molecules, pathogens and commensal micro-organisms, requires the ability to discriminate between them and to express appropriate responses, i.e. the increased complexity of recognition systems requires increased complexity of the mechanisms for assessing 'quality' of antigen.

Clearly, B and T lymphocytes themselves are targets for mechanisms enforcing tolerance, since they express the rearranged antigen-specific receptors. However, although B-cells recognise structural elements of antigen directly, T-cells first require previous processing of the antigen by antigen-presenting cells. Thus, the antigen-presenting cell is also a target for tolerance induction.

Interestingly, this problem may have been solved more than once in evolution. Until recently, the adaptive immune system of the jawed vertebrates was considered unique. However, recent studies have identified other possible adaptive immune systems: in jawless fish, dependent on rearranging leucine-rich repeat molecules; in snails and protochordates, both dependent on hypermutating Ig-like domains (Flajnik \& Du Pasquier, 2004). These findings, if confirmed, strongly suggest polyphyletic evolution of adaptive recognition systems and, therefore, of mechanisms for tolerance induction. However, these mechanisms are likely to be radically different from those in higher vertebrates.

\section{Structure of the immune system}

\section{Evolution}

The earliest animals in which the vertebrate adaptive immune system evolved are likely to be related to the armoured jawed fish, the placoderms, about $400 \times 10^{6}$ years ago. The most distant extant group possessing a vertebrate adaptive immune system are the cartilaginous fish, the sharks, skates and rays. In these animals relatively little of the gross structure of the immune system is recognisable. Those parts that are recognisable include the thymus, in which T lymphocyte tolerance to self antigens is generated, and the intestine (Zapata \& Cooper, 1990). The presence of an intestinal immune system in these species indicates that the need for expression of active immune responses and 
tolerance at mucosal surfaces was one of the earliest imperatives for the newly-evolved recognition mechanisms.

\section{The mucosal immune system of mammals}

Work over several decades has demonstrated an extent of separation between those components of the immune system dealing with antigen at mucosal surfaces and those involved with systemic antigen (Phillips-Quagliata, 2002). Thus, a range of studies have demonstrated that lymphocytes isolated from mucosal tissues, then labelled and re-injected show a preference for homing back to mucosal sites; similarly, systemically-derived lymphocytes re-home to systemic lymphoid tissue. This preferential homing is dependent on the expression of tissue-specific receptors on lymphocytes that either bind to differentially-expressed adhesion molecules on endothelial cells, or are activated by chemokines specific for the target tissue (Butcher et al. 1999; Kunkel \& Butcher, 2002). The activation of T-cells in specific sites by presenting cells that bear cognate antigen 'imprints' selective homing by triggering the expression of the appropriate receptors (Stagg et al. 2002). Importantly, this phenomenon is not absolute; direct labelling studies and indirect studies of tissue distribution of adhesion molecules suggest some cross-over between homing in the systemic and mucosal immune systems (McDermott \& Bienenstock, 1979).

In mammals one of the defining characteristics of the mucosal immune system is the production of secretory antibody of the $\operatorname{IgA}$ class. Specialisations of $\operatorname{IgA}$ as a mucosal Ig include basal-to-apical transport by epithelial cells (mediated by the polymeric Ig receptor), resistance to lumen proteolytic activity and binding to mucus (Johansen et al. 2001). Functionally, IgA does not appear to directly influence the composition of the lumen commensal microflora (Sait et al. 2003); however, it does act to prevent antigens and micro-organisms from penetrating the epithelium (Macpherson \& Uhr, 2004b) and to pump antigens that have penetrated by binding before being transported into the intestinal lumen across epithelial cells (Brown et al. 1984).

The organised lymphoid system. Organised lymphoid tissue in the mammals is defined by the presence of large numbers of different types of leucocytes clearly separated into distinct zones. Further, these zones should include distinct B-lymphocyte areas (the follicles) and T-cell zones, allowing functional specialisation within the tissues (Brandtzaeg \& Pabst, 2004). The term mucosal-associated lymphoid tissues is reserved for this organised lymphoid tissue, which, in the intestine, includes the Peyer's patches in the wall of the intestine, with direct access to the lumen contents, and the mesenteric lymph nodes supplied by the afferent lymphatics from the intestinal mucosal and Peyer's patches (gut-associated lymphoid tissue).

The diffuse lymphoid system. Outside the organised lymphoid tissues, the intestinal epithelium and mucosa are also heavily infiltrated with leucocytes, primarily lymphocytes, macrophages and dendritic cells. The term 'controlled inflammation' is frequently used to describe this leucocytic infiltration. However, inflammation is characterised by changes in capilliary endothelial cell function and expression of adhesion molecules that have not been described in normal intestine. Rather, the cellular infiltrate shows a distinct level of organisation, CD8+ $\mathrm{T}$-cells predominating in the epithelium, CD4+ T-cells in the villus lamina propria and, in the pig, plasma cells around the crypts (Vega-Lopez et al. 1993; Wilson et al. 1996). Although technically not part of the gut-associated lymphoid tissue, the diffuse architecture of the mucosa does show a level of organisation consistent with specialisation of function, and this organisation is particularly marked in the pig.

\section{Mucosal tolerance}

The demonstration of immunological tolerance to harmless fed protein is usually performed using a so-called 'oral tolerance' protocol. In these experiments groups of mice are fed normal diet or one that includes a novel protein antigen. After a period of time both groups are immunised with the homologous antigen and the previously-fed group mount markedly reduced responses, indicating specific immunological tolerance (Challacombe \& Tomasi, 1980; Bailey et al. 1993). However, there are major problems with the interpretation of such experiments. First, the challenge is systemic rather than mucosal and, formally, does not demonstrate tolerance in the mucosal immune system. Some strains and some experimental manipulations produce mice that tolerise poorly to novel fed antigens (by systemic challenge) but do not appear to express aberrant responses to their normal diet, indicating active systemic responses with mucosal tolerance (Stokes et al. 1983b; Fujihashi et al. 2001; Spahn et al. 2001). Second, oral tolerance may be associated with mucosal secretion of $\operatorname{IgA}$ antibody, indicating systemic tolerance with active local immune responses (Challacombe \& Tomasi, 1980).

Tolerance to antigens associated with commensal flora has been more difficult to demonstrate and has provided conflicting results. Duchmann et al. $(1995,1996)$ have shown that T-cells from mice or man respond poorly to antigens of autologous flora compared with those of heterologous flora and have concluded that individuals are tolerant to their own flora (this protocol is not, formally, an oral-tolerance protocol). Further studies have indicated that this tolerance is abrogated in inflammatory bowel disease. This observation has led to a very productive line of research that has examined the role of immune responses to commensal microbial flora in a range of models of colitis in mouse and man. However, the concept has been challenged by the demonstration that systemic immunisation with commensal bacteria can elicit an active response in precolonised animals, in which local $\operatorname{IgA}$ antibody can also be demonstrated. In this model systemic responses to commensal flora do not occur because bacteria are confined to the mucosal immune system (immunological ignorance; Macpherson \& Uhr, 2004a). Thus, the data on responses to lumen flora are conflicting at the moment. 


\section{Mechanisms}

Work over several decades has begun to address the mechanisms involved in maintaining tolerance. Clearly, immunological ignorance is one possibility. However, antigen derived from fed protein is relatively easy to demonstrate in serum, indicating that the systemic immune system is exposed to food antigens.

Anergy or deletion of specific T-cell clones has also been proposed as an important mechanism (Mowat et al. 2004). Such functional inactivation can occur when antigen-naïve T-cells recognise antigen on inappropriate presenting cells. Dendritic cells, the primary cell responsible for presentation to naïve T-cells, can trigger active clonal expansion or functional inactivation, depending on the signals available from the environment. Dendritic cells express a wide range of pattern-recognition receptors that can signal the presence of pathogen in addition to providing antigen-specific signals. The ability of dendritic cells to switch naïve T-cells into tolerance or active response pathways has led to the proposal that the interaction between dendritic cells and naïve T-cells in the initial primary response to novel mucosal antigen may determine active responses or tolerance (Mowat et al. 2004). Susceptibility of T-cells to apoptosis has been demonstrated in mucosal tissues, but importantly in primed effector T-cells in the intestinal epithelium and lamina propria $(\mathrm{Bu}$ et al. 2001; Levine \& Fiocchi, 2001). Thus, an alternative proposal is that antigen-non-specific signals to mucosal effector T-cells determine the outcome of antigen recognition (either clonal expansion and expression of active immune responses or clonal deletion and tolerance; Bailey et al. 2005). The difference between these two models lies in the cell responsible for integrating environmental signals through pattern-recognition receptors, adhesion molecules and cytokine receptors: in the first model dendritic cells integrate the signals and determine the response of naive T-cells; in the second model effector T-cells directly integrate signals themselves during antigen recognition on a range of presenting cells.

Recently, the role of regulatory T-cells in maintaining tolerance has been extensively described. The regulatory T-cell type involved in maintaining mucosal tolerance appears to be peripherally generated, requires antigen for expression of function, but expresses regulation in an antigen-independent way (Samsom, 2004). Regulation is expressed through secretion of the cytokines IL-10 and/or transforming growth factor $\beta$ (TGF $\beta$ ), although there is evidence for a requirement for cell-cell interactions. These two cytokines (IL-10 and TGF $\beta$ ) may also act to promote differentiation of uncommitted T-cells into regulatory $\mathrm{T}$ cells, providing a negative (regulatory) feedback loop (Peng et al. 2004).

TGF $\beta$ in particular may play an important role, since in addition to being produced by regulatory cells it is also produced by a range of other cell types in the intestine, including epithelial cells, endothelial cells and myofibroblasts. Receptors are expressed on a wide range of target cell types, and signalling through TGF $\beta$ RII is essential for switching to IgA production at mucosal sites (Borsutzky et al. 2004). Thus, TGF $\beta$ is a likely candidate for generating tolerance and $\operatorname{IgA}$ responses concurrently, although mucosal stromal cells may be a more important source than regulatory $\mathrm{T}$-cells.

\section{Sites}

Primary responses to antigen clearly occur in the organised lymphoid tissues and this factor, together with the known role of dendritic cell presentation to naïve T-cells, has led to the inference that tolerance is determined in the Peyer's patches and mesenteric lymph nodes during primary recognition of novel antigens. However, given the level of organisation of the diffuse mucosal lymphoid architecture, it is also entirely possible that tolerance is also determined in this site (Bailey et al. 2005). In fact, several studies have demonstrated that the phenomenon of tolerance is preceded by an active immune response, suggesting that tolerance is determined at the effector stage, rather than the inductive stage (Stokes et al. 1983a; Bailey et al. 1993).

\section{The immune system of neonates}

Two important periods of maximum exposure to novel antigens occur in the young animal, immediately after birth and at weaning. In both cases the antigenic composition of the intestinal contents can shift suddenly, as a result of novel diet and of colonisation by novel strains and species of bacteria. In newborn mammals colonisation with environmental bacteria begins soon after birth, exposing the neonate to a range of microbial antigens and also to non-specific microbial products capable of triggering pattern-recognition receptors. Changes in lifestyles of man, and husbandry of other mammals, have resulted in weaning becoming much more abrupt than previously in evolution, increasing the number of antigens that must be simultaneously evaluated by growing animals. Novel antigens at weaning will include those derived from food, but also those from the shift in intestinal bacterial populations occurring as a result of dietary change. Thus, birth and weaning are likely to represent hazard and critical control points in the development of appropriate responses to pathogens and harmless dietary and commensal antigens, i.e. factors that compromise the ability of the mucosal immune system to make appropriate assessments of the 'quality' of antigens, or to select appropriate pathways of active immune response or tolerance, are likely to have much greater effects on animals at these time points.

\section{The mucosal immune system in neonatal animals}

There is considerable evidence to indicate that the immune system of neonates is relatively undeveloped compared with that of adults, particularly the mucosal immune system (Vega-Lopez et al. 1995). Consistent with this observation, newborn animals mount poor responses to antigen, and can often be tolerised by doses of antigen that trigger active responses in adults (Adkins et al. 2004). Further, they are difficult to tolerise orally (Peng et al. 1989), indicating that the major functions of the systemic and 
mucosal immune systems are defective at birth. Together, these factors probably help to prevent inappropriate immune responses to the range of novel antigens presented to the newborn animal.

Other factors will also contribute, specifically factors in maternal milk. Passive transfer of maternally-derived antibody clearly has a protective effect and is likely to provide 'cover' during a period when the neonatal immune system is insufficiently developed to respond actively. However, maternal colostrum also contains high levels of TGF $\beta$, which gradually decreases in milk during lactation (Xu et al. 1999). The role of TGF $\beta$ in regulating mucosal immune responses has been discussed, and there is evidence that maternally-derived TGF $\beta$ may accelerate the switch to secretion of $\operatorname{IgA}$ antibody to common food proteins and protect against the early development of atopic disease in human infants (Kalliomaki et al. 1999; Ogawa et al. 2004). Supplementation of food with one isoform, TGF $\beta 2$, can ameliorate experimental colitis in mice $(\mathrm{Oz}$ et al. 2004) and, interestingly, this isoform may be reduced in cow's milk compared with human milk (Chun et al. 2004).

At birth a combination of these factors may prevent both expression of active immune responses and development of tolerance. However, colonisation by intestinal flora expands the mucosal immune system in antigen-specific and non-specific ways. Within days after birth the organised lymphoid tissues expand and antigen-presenting cells and T-cells begin to infiltrate the intestine. Current studies using piglets (C Harris, K Haverson, $\mathrm{P}$ Jones, C Inman, M Bailey, B Miller and CR Stokes, unpublished results) indicate that the $\mathrm{T}$-cells that enter the intestinal mucosa at this time express activation-associated molecules, consistent with initial active responses to antigen. It seems likely that these T-cells are controlled, and their differentiation to regulatory-regulated phenotype promoted, by the presence of TGF $\beta$ derived from stromal cells and from milk. The activated phenotype disappears quickly, and 3 weeks after birth the CD4+ T-cells in the intestine are comparable with those present in adults. The CD8 + T-cell population (primarily in the epithelium) takes a further 2 weeks to mature.

In germ-free animals very little of this development of the mucosal immune system occurs (Rothkotter et al. 1991; Butler et al. 2000) and such animals remain relatively poor at generating oral tolerance (Prioult et al. 2003). Microbial colonisation of the intestine drives the development of the immune system in several ways, and much of it is antigen-specific, i.e. representing a specific response to bacterial antigens. However, much appears to be antigen-non-specific, presumably driven by ligation of pattern-recognition receptors by non-specific microbial products (Talham et al. 1999; Butler et al. 2000). Thus, development of an efficient mucosal immune system appears to be dependent on colonisation by commensal and pathogenic microbial flora.

These experimental observations have implications for the so-called 'hygiene hypothesis', which proposes that the increasing prevalence of atopy in man is attributable to decreasing contact with the microbial flora with which man's immune systems have evolved (Helm \& Burks,
2000). There is strong epidemiological evidence for this hypothesis in man. Initially, the observation that the earliest immune responses generated by neonates tend to be of the T-helper (TH) (antibody, IgE) type 2 led to the suggestion that the balance between Th1 (cellular) and Th2 responses in neonates is skewed towards Th2, resulting in IgE-type responses to environmental antigens. Colonisation by microbial flora was believed to drive expansion of Th1-type systems resulting in 'balanced' (i.e. non-allergic) responses (Holt et al. 1997). However, recent observations that parasite-infected children are less susceptible to atopy are not consistent with this model, since parasites tend to drive Th2 responses (van den Biggelaar et al. 2000). More consistent is the idea that colonisation results in balanced expansion of effector and regulatory arms of the immune system, such that responses to mucosal antigen can be either active or tolerogenic (Bailey et al. 2001).

It is proposed that under normal conditions the ability of the mucosal immune system to mount active responses to foreign antigens develops simultaneously with the ability to control and regulate such responses. Problems arise when one or other arm of the immune system develops inappropriately, resulting in inappropriate effector responses to harmless food proteins (allergy) or inadequate responses to pathogen (disease susceptibility). This hypothesis carries important implications for assessment of manipulation on the development of the neonatal immune system. Specifically, assessment only of the ability of the mucosal immune system to mount active immune responses is not a safe way of evaluating nutritional intervention: the impact of such interventions on tolerance induction also needs to be assessed.

\section{The mucosal immune system at weaning}

In contrast to birth the antigenic challenge at weaning occurs when the mucosal immune system has developed to the point of making active immune responses. In addition, the immunoregulatory and immunoprotective components of maternal milk are both removed. Both in man and in piglets antibody to fed proteins can be detected after weaning, indicating active immune responses to fed proteins. Despite this initial active response to fed proteins some form of tolerance is ultimately achieved, although this transition may take some time (Miller et al. 1994; Bailey et al. 2004). In rodents weaning has been associated with changes in activation status and numbers of leucocytes in the intestinal mucosa (Manzano et al. 2002). However, in weaned piglets the changes are much more subtle, possibly representing the difference in preweaning microbial challenge between rodents in experimental specific-pathogen-free facilities and young piglets (Bianchi et al. 1992; C Harris, K Haverson, P Jones, C Inman, M Bailey, B Miller and CR Stokes, unpublished results). TGF $\beta 1$ does decrease transiently in enterocytes, while leucocyte TGF $\beta 1$ increases, suggesting complex shifts in the regulatory environment. 


\section{Conclusions}

The mucosal immune system clearly serves two functions, to mount active responses against pathogens and to mount tolerance against harmless food and commensal bacterial antigens. Both functions involve active processes and, therefore, carry nutritional costs. Critical to the function of the intestine is the ability to assess the 'quality' of antigens and to respond appropriately; the costs of inappropriate decisions are allergy or chronic susceptibility to disease.

Increasing evidence suggests that intestinal homeostasis requires the mucosal immune system of the neonate to develop as a balance between regulatory and effector function, i.e. that the regulatory influences must expand as effector function expands. Birth and weaning are likely to represent hazard and critical control points in this process. In the newborn animal factors in maternal milk may regulate responses (TGF $\beta$ ) as well as providing passive effector molecules (Ig). At weaning these factors are removed and there is evidence that the balance may be disturbed. The magnitude and severity of this response is likely to be dependent on the extent to which the effector and regulatory arms of the immune system have been expanded during the preweaning period.

\section{References}

Adkins B, Jones M, Bu YR \& Levy RB (2004) Neonatal tolerance revisited again: specific CTL priming in mouse neonates exposed to small numbers of semi- or fully allogeneic spleen cells. European Journal of Immunology 34, 1901-1909.

Bailey M, Haverson K, Inman C, Harris C, Jones P, Corfield G, Miller B \& Stokes C (2005) The influence of environment on development of the mucosal immune system. Veterinary Immunology and Immunopathology (In the Press).

Bailey M, Haverson K, Miller B, Jones P, Sola I, Enjuanes L \& Stokes CR (2004) Effects of infection with transmissible gastroenteritis virus on concomitant immune responses to dietary and injected antigens. Clinical and Diagnostic Laboratory Immunology 11, 337-343.

Bailey M, Miller BG, Telemo E, Stokes CR \& Bourne FJ (1993) Specific immunological unresponsiveness following active primary responses to proteins in the weaning diet of pigs. International Archives of Allergy and Applied Immunology 101, 266-271.

Bailey M, Plunkett FJ, Rothkotter H-J, Vega-Lopez MA, Haverson K \& Stokes CR (2001) Regulation of mucosal immune responses in effector sites. Proceedings of the Nutrition Society 60, 1-8.

Bianchi ATJ, Zwart RJ, Jeurissen SHM \& Moonen-Leusen HWM (1992) Development of the B-cell and T-cell compartments in porcine lymphoid organs from birth to adult life - an immunohistological approach. Veterinary Immunology and Immunopathology 33, 201-221.

Beutler B \& Rehli M (2002) Evolution of the TIR, tolls and TLRs: Functional inferences from computational biology. Current Topics in Microbiology and Immunology 270, 1-21.

Borsutzky S, Cazac BB, Roes J \& Guzman CA (2004) TGF-beta receptor signaling is critical for mucosal $\operatorname{IgA}$ responses. Journal of Immunology 173, 3305-3309.

Brandtzaeg P \& Pabst R (2004) Let's go mucosal: communication on slippery ground. Trends in Immunology 25, 570-577.
Brown TA, Russell MW \& Mestecky J (1984) Elimination of intestinally absorbed antigen into the bile by IgA. Journal of Immunology 132, 780-782.

Bu P, Keshavarzian A, Stone DD, Liu JZ, Le PT, Fisher S \& Qiao L (2001) Apoptosis: One of the mechanisms that maintains unresponsiveness of the intestinal mucosal immune system. Journal of Immunology 166, 6399-6403.

Butcher EC, Williams M, Youngman K, Rott L \& Briskin M (1999) Lymphocyte trafficking and regional immunity. Advances in Immunology 72, 209-253.

Butler JE, Sun J, Weber P, Navarro P \& Francis D (2000) Antibody repertoire development in fetal and newborn piglets. III Colonization of the gastrointestinal tract selectively diversifies the preimmune repertoire in mucosal lymphoid tissues. Immunology 100, 119-130.

Challacombe SJ \& Tomasi TB (1980) Systemic tolerance and secretory immunity after oral immunization. Journal of Experimental Medicine 152, 1459-1472.

Chun SK, Nam MS, Goh JS, Kim WS, Han YH \& Kim PH (2004) Kinetics and biological function of transforming growth factor-beta isoforms in bovine and human colostrum. Journal of Microbiology and Biotechnology 14, 1267-1274.

Duchmann R, Kaiser I, Hermann E, Mayet W, Ewe K \& zum Buschenfelde K-HM (1995) Tolerance exists towards resident intestinal flora but is broken in active inflammatory bowel disease (IBD). Clinical and Experimental Immunology 102, $448-455$.

Duchmann R, Schmitt E, Knolle P, zum Buschenfelde KHM \& Neurath M (1996) Tolerance towards resident intestinal flora in mice is abrogated in experimental colitis and restored by treatment with interleukin-10 or antibodies to interleukin-12. European Journal of Immunology 26, 934-938.

Flajnik MF \& Du Pasquier L (2004) Evolution of innate and adaptive immunity: can we draw a line? Trends in Immunology 25, 640-644.

Fujihashi K, Dohi T, Rennert PD, Yamamoto M, Koga T, Kiyono H \& McGhee JR (2001) Peyer's patches are required for oral tolerance to proteins. Proceedings of the National Academy of Sciences USA 98, 3310.

Helm RM \& Burks AW (2000) Mechanisms of food allergy. Current Opinion in Immunology 12, 647-653.

Holt PG, Sly PD \& Bjorksten B (1997) Atopic versus infectious diseases in childhood: a question of balance? Pediatric Allergy and Immunology 8, 53-58.

Johansen FE, Braathen R \& Brandtzaeg P (2001) The J chain is essential for polymeric Ig receptor-mediated epithelial transport of IgA. Journal of Immunology 167, 5185-5192.

Kalliomaki M, Ouwehand A, Arvilommi H, Kero P \& Isolauri E (1999) Transforming growth factor-beta in breast milk: A potential regulator of atopic disease at an early age. Journal of Allergy and Clinical Immunology 104, 1251-1257.

Kunkel EJ \& Butcher EC (2002) Chemokines and the tissuespecific migration of lymphocytes. Immunity 16, 1-4.

Levine AD \& Fiocchi C (2001) Regulation of life and death in lamina propria T cells. Seminars in Immunology 13, 195-199.

Litman GF, Anderson MK \& Rast J (1999) Evolution of antigen binding receptors. Annual Review of Immunology 17, 109-147.

Luo C \& Zheng L (2000) Independent evolution of Toll and related genes in insects and mammals. Immunogenetics 51, 92-98.

McDermott MR \& Bienenstock J (1979) Evidence for a common mucosal immunological system. 1. Migration of B immunoblasts into intestinal, respiratory, and genital tissues. Journal of Immunology 122, 1892-1898.

MacPherson AJ \& Uhr T (2004a) Compartmentalization of the mucosal immune responses to commensal intestinal bacteria Annals of the New York Academy of Sciences 1029, 36-43. 
MacPherson AJ \& Uhr T (2004b) Induction of protective IgA by intestinal dendritic cells carrying commensal bacteria. Science 303, 1662-1665.

Manzano M, Abadia-Molina AC, Garcia-Olivares E, Gil A \& Rueda R (2002) Absolute counts and distribution of lymphocyte subsets in small intestine of BALB/c mice change during weaning. Journal of Nutrition 132, 2757-2762.

Miller BG, Whittemore CT, Stokes CR \& Telemo E (1994) The effect of delayed weaning on the development of oral tolerance to soybean protein in pigs. British Journal of Nutrition 71, 615-625.

Mowat AM, Parker LA, Beacock-Sharp H, Millington OR \& Chirdo F (2004) Oral tolerance: Overview and historical perspectives. Annals of the New York Academy of Sciences 1029, $1-8$.

Ogawa J, Sasahara A, Yoshida T, Sira MM, Futatani T, Kanegane $\mathrm{H} \&$ Miyawaki T (2004) Role of transforming growth factorbeta in breast milk for initiation of IgA production in newborn infants. Early Human Development 77, 67-75.

Oz HS, Ray M, Chen TS \& McClain CJ (2004) Efficacy of a transforming growth factor 82 containing nutritional support formula in a murine model of inflammatory bowel disease. Journal of the American College of Nutrition 23, 220-226.

Peng HJ, Turner MW \& Strobel S (1989) Failure to induce oral tolerance to protein antigens in neonatal mice can be corrected by transfer of adult spleen-cells. Pediatric Research 26, 486-490.

Peng YF, Laouar Y, Li MO, Green EA \& Flavell RA (2004) TGF-beta regulates in vivo expansion of Foxp3-expressing $\mathrm{CD} 4(+) \mathrm{CD} 25(+)$ regulatory $\mathrm{T}$ cells responsible for protection against diabetes. Proceedings of the National Academy of Sciences USA 101, 4572-4577.

Phillips-Quagliata JM (2002) Adhesion molecules and circulation and differentiation of lymphocytes in GALT and mammary glands. In Food Allergy and Intolerance, chapter 4 [J Brostoff and SJ Challacombe, editors]. London: Saunders.

Prioult G, Fliss I \& Pecquet S (2003) Effect of probiotic bacteria on induction and maintenance of oral tolerance to betalactoglobulin in gnotobiotic mice. Clinical and Diagnostic Laboratory Immunology 10, 787-792.

Rothkotter HJ, Ulbrich H \& Pabst R (1991) The postnataldevelopment of gut lamina propria lymphocytes - number, proliferation, and T-cell and B-cell subsets in conventional and germ-free pigs. Pediatric Research 29, 237-242.

Sait L, Galic M, Strugnell RA \& Janssen PH (2003) Secretory antibodies do not affect the composition of the bacterial microbiota in the terminal ileum of 10-week-old mice. Applied and Environmental Microbiology 69, 2100-2109.

Samsom JN (2004) Regulation of antigen-specific regulatory T-cell induction via nasal and oral mucosa. Critical Reviews in Immunology 24, 157-177.

Schroder HC, Ushijima H, Krasko A, Gamulin V, Thakur NL, Diehl-Seifert B, Muller IM \& Muller WEG (2003) Emergence and disappearance of an immune molecule, an antimicrobial lectin, in basal Metazoa - A tachylectin-related protein in the sponge Suberites domuncula. Journal of Biological Chemistry 278, 32810-32817.

Spahn TW, Fontana A, Faria AMC, Slavin AJ, Eugster HP, Zhang XM, Koni PA, Ruddle NH, Flavell RA, Rennert PD \& Weiner HL (2001) Induction of oral tolerance to cellular immune responses in the absence of Peyer's patches. European Journal of Immunology 31, 1278.

Stagg AJ, Kamm MA \& Knight SC (2002) Intestinal dendritic cells increase $\mathrm{T}$ cell expression of alpha 4 beta 7 integrin. European Journal of Immunology 32, 1445-1454.

Stokes CR, Newby TJ \& Bourne FJ (1983a) The influence of oral immunisation on local and systemic immune responses to heterologous antigens. Clinical and Experimental Immunology 52, 399-406.

Stokes CR, Swarbrick ET \& Soothill JF (1983b) Genetic differences in immune exclusion and partial tolerance to ingested antigens. Clinical and Experimental Immunology 52, 678-684.

Talham GL, Jiang HQ, Bos NA \& Cebra JJ (1999) Segmented filamentous bacteria are potent stimuli of a physiologically normal state of the murine gut mucosal immune system. Infection and Immunity 67, 1992-2000.

Teillet F, Dublet B, Andrieu JP, Gaboriaud C, Arland GJ \& Thielens NM (2005) The two major oligomeric forms of human mannan-binding lectin: Chemical characterization, carbohydrate-binding properties, and interaction with MBL-associated serine proteases. Journal of Immunology 174 2870-2877.

van den Biggelaar AHJ, van Ree R, Rodrigues LC, Lell B, Deelder AM, Kremsner PG \& Yazdanbakhsh M (2000) Decreased atopy in children infected with Schistosoma haematobium: a role for parasite-induced interleukin-10. Lancet 356, 1723-1727.

Vega-Lopez MA, Bailey M, Telemo E \& Stokes CR (1995) Effect of early weaning on the development of immune cells in the pig small-intestine. Veterinary Immunology and Immunopathology 44, 319-327.

Vega-Lopez MA, Telemo E, Bailey M, Stevens K \& Stokes CR (1993) Immune cell distribution in the small-intestine of the pig - immunohistological evidence for an organized compartmentalization in the lamina propria. Veterinary Immunology and Immunopathology 37, 49-60.

Wilson AD, Haverson K, Southgate K, Bland PW, Stokes CR \& Bailey M (1996) Expression of major histocompatibility complex class II antigens on normal porcine intestinal endothelium. Immunology 88, 98-103.

Xu RJ, Doan QC \& Regester GO (1999) Detection and characterisation of transforming growth factor-beta in porcine colostrum. Biology of the Neonate 75, 59-64.

Zapata AG \& Cooper EL (1990) The Immune System: Comparative Histophysiology. Chichester, West Sussex: John Wiley. 